\title{
Sexual health during menopause - counselling with an approach to emotional regulation on sexual satisfaction
}

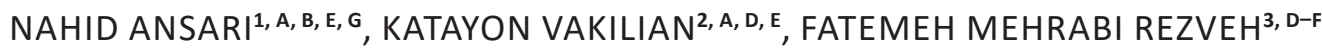 \\ ORCID ID: 0000-0002-6035-0796 \\ ${ }^{1}$ Arak University of Medical Sciences, Arak, Iran \\ ${ }^{2}$ School of Medicine, Traditional and Complementary Medicine Research Center (TCMRC), Arak University \\ of Medical Sciences, Arak, Iran \\ ${ }^{3}$ Azad University of Arak, Arak, Iran
}

A - Study Design, B - Data Collection, C - Statistical Analysis, D - Data Interpretation, E - Manuscript Preparation, F - Literature Search, G - Funds Collection

Summary Background and objectives. One of the concerns of menopause is the decrease of sexual desire or satisfaction. Therefore, the present study was conducted to investigate the effect of group counselling with an approach to emotional regulation on sexual satisfaction of postmenopausal women.

Material and methods. The present research was a randomised educational trial study and was conducted in 2017 in Khomein in the Markazi province Iran on postmenopausal women visiting a health centre specialising in physical health assessment for elderly women. The 200 participants who chose to participate, after obtaining written consent, completed questionnaires. Finally, 40 eligible participants were included in the study based on lower score 75 in sexual satisfaction questionnaire and lower score 270 in emotional quotient questionnaire. 20 individuals were allocated to the intervention group (emotional regulation), and 20 individuals in control group by by two arms block. The intervention group attended 8 emotional regulation counselling sessions, and the control group received the routine care. The sexual satisfaction of postmenopausal women was measured before and one month after the intervention using the Larson sexual satisfaction questionnaire and Bar-On's emotional quotient questionnaire, respectively. The data was analysed through SPSS software version 23, as well as the Mann-Whitney $U$ test, $t$-test, Wilcoxon Sign test and chi-square test.

Results. The mean sexual satisfaction score $(78.3 \pm 14.8)$ increased after the intervention of emotional regulation in counselling group compared to the control group ( $p>0.05$ ), but this wasn't significant.

Conclusions. Although emotional regulation can be considered as an appropriate method to improve sexual satisfaction in these women, more study should be done in the future.

Key words: health, sexual health, menopause, orgasm.

Ansari N, Vakilian K, Rezveh FM. Sexual health during menopause - counselling with an approach to emotional regulation on sexual satisfaction. Fam Med Prim Care Rev 2019; 21(4): 318-323, doi: https://doi.org/10.5114/fmpcr.2019.90159.

\section{Background}

Menopause is a natural biological process that affects women's lives personally, socially and culturally and is of considerable importance as one of the hygiene issues in reproductive health [1]. Today, the elderly live longer and are healthier than before, and women spend about one third of their life in menopause. The population growth of the elderly and hygiene problems after menopause have increased the need to understand the changes and problems of this age and create new expectations in hygiene issues in order to experience a successful old age $[2,3]$. As the number of older people is increasing in developed countries, community attitudes are changing in old-age lifestyles, as well as behaviours. One of the changes in behaviours in old-age couples that should be noticed by health services and health providers is sexual issues [4].

Studies indicate that a strong relationship exist between normal sexual relationship and quality of life in old age. Women who attach little importance to their sexual relationships and engage in less sexual activity after menopause experience more vaginal dryness and atrophy, eventually experiencing painful sexual intercourse and becoming more isolated than other women $[5,6]$.

Sexual satisfaction is a multidimensional concept that includes the emotional and physiological aspects of a sexual relationship. Sexual satisfaction is not only physical pleasure, but it includes all the positive emotions after sexual relationship [7]. Lawrance and Byers defined sexual satisfaction as an emotional response derived from individuals' mental evaluation of the positive and negative dimensions of a sexual relationship [8]. Dissatisfaction with sexual relationships leads to major problems in couples' relationships, including hatred towards their spouse, annoyance, jealousy, feelings of vengeance, embarrassment and being neglected and lack of self-confidence. These problems worsen with tensions and disagreements between couples, widen their emotional gap and consequently lead to dissatisfaction [9].

It seems that many Iranian couples suffer from sexual dissatisfaction; however, they feel ashamed to talk about it and are not aware of its effect on marital dissatisfaction and, therefore, on dissatisfaction with life $[10,11]$.

The ability to have good sexual relationships and satisfaction at any age depends on some teachable and learnable skills $[12,13]$.

One of the approaches that have attracted attention nowadays is emotional regulation, since it is perfectly capable of solving relationship problems [14].

Emotional regulation refers to the acceptance, understanding and management of emotions [14]. Generally, emotional regulation can be considered as a process by which the person influences the form of their current emotion. This process determines how the person experiences and expresses their emo- 
tions [15]. Specifically, emotional regulation as a special form of self-regulation refers to acts changing or moderating a particular emotion. In fact, emotional regulation is the answer to the questions of whether the person can identify or describe their emotions and explain the intensity of experiencing them or whether the person intends to express or suppress the emotions. By understanding the emotions skilfully, people can face current problems and avoid future ones [16, 17]. Since sexual satisfaction includes an emotional dimension, and emotional regulation deals with this dimension, it seems that by improving and developing emotional regulation skills, sexual satisfaction can be enhanced [18].

\section{Objectives}

Descriptive-correlational studies have investigated sexual satisfaction along with emotional regulation $[18,19]$; however, there are few interventional studies in this field.

Therefore, in this study, the effect of group counselling with an approach to emotional regulation on sexual satisfaction of postmenopausal women was investigated.

\section{Material and methods}

\section{Study design}

This research is a randomised educational trial study and was conducted on 40 postmenopausal married women.

\section{Setting}

This study was carried out at the Khomein health centre between July to October 2017 in Khomein city in the Markazi province of Iran. Considering the standard deviation sexual satisfaction $\left(s_{1}=1.7, s_{2}=1.5\right)$ and maximum error of $d=2.5$ and confidence level of $95 \%, 20$ samples were entered into the study. They entered the research and divided in two groups ( $A$ and $B$ ) based on a quadruple randomized block. Block randomization was done by Excel soft ware.

Eight 90-minute group counselling sessions with an approach to emotional regulation were held for group " $A$ ", while group B did not receive counselling. Sessions were held by an MSc student in midwifery counselling who had certification in emotional regulation approach.

The protocol of group counselling sessions with an approach to emotional regulation for the intervention group was as follows:

Session one: welcoming, introduction, explaining the group rules, arranging the dates and hours of holding the sessions, defining sexual satisfaction and explaining the consequences of sexual dissatisfaction, defining menopause and the sexual problems of this period and defining the emotional quotient and its components (self-awareness, self-regulation, motivation, empathy and social relationship control).

Session two: a summary of previous session, answering the questions, familiarising the members with the symptoms of defects in emotion, introducing emotional quotient skills and training the emotion (first skill).

Home assignments were:

1. Reviewing of emotional situations for the next week.

2. Record in a table how you felt and behaved in different situations, especially sex.

Session three: a summary of the previous session, pursuing the assignments, answering the questions, overcoming the obstacles to normal emotions (second emotional quotient skill) and reducing physical harm (third emotional quotient skill). the familiarity of the members with all-round thinking and consequently balancing between emotions and thoughts using group scenarios, and characterizing the harmful behaviours, criticism negative thoughts in the scenarios presented in the groups, identifying self-harm behaviours in practices.

Home assignments were:

1. Providing a list of your beliefs and thoughts about sex during menopause.

2. Reviewing one's thoughts, emotions and behaviours in sexual situations (written assignment).

\section{Session four:}

A summary of previous session, pursuing the assignments, answering the questions, the familiarity of the members with all-round thinking and the consequently balancing between emotions and thoughts using group scenarios (fourth skill).

Home assignments were:

1. Providing a list of negative thoughts about sex and altering a positive through for them.

2. Reviewing the disturbing emotion during sex using the balancing skill between thought and feeling.

Session five: a summary of the previous session, pursuing the assignments, answering the questions, positive illustration, methods of increasing positive emotions (fifth skill) and conscious attention to emotions without judging them (sixth skill), knowledge of the members by the method of conscious attention to emotions with practical exercises in the session.

Home assignments were:

1. Practicing favourite sexual imaging with details at home.

2. Preparing a list of daily joyful activities.

Session six: a summary of the previous session, pursuing the assignments, answering the questions, increasing the encounter with emotions (seventh skill), teaching the use of the five senses, increasing the encounter with emotions and breathing technique education.

Home assignments were:

1. Using the five senses in sex.

2. Confronting disturbing emotions and thoughts in sex through a gentle breathing technique.

Session seven: a summary of the previous session, pursuing the assignments, answering the questions and actions opposite to emotions. Practicing the emotion-opposite action in the scenarios presented in the sessions.

Home assignments were: continuing the home assignments from before.

Session eight: a summary of the previous session, pursuing the assignments, answering the questions, conclusion, vote of thanks, farewell and making a date to distribute the questionnaires for one month later. Practicing the emotion-opposite action in the scenarios presented in the sessions.

Home assignment was recording emotions and emotion-based behaviour and then writing an opposite action which can replace it.

In this study, all of the women followed the 8 sessions. One month after intervention two groups evaluated for sexual satisfaction and emotional quotient. Upon completing the study, and after assessment of sexual satisfaction, a pamphlet on the contents of sessions was distributed to the control group.

\section{Participants}

300 postmenopausal married women were assigned in this health center. This center visited postmenopausal for physical health. Researcher contacted to them by telephone. Finally 200 invited to the research. According to the evaluation of inclusion criteria, 40 eligible women among the 200 women who intended to participate in the study after filling in a written consent form were included in the study. Finally, a total of 40 eligible women were selected for the study using convenience sampling (Figure 1). 


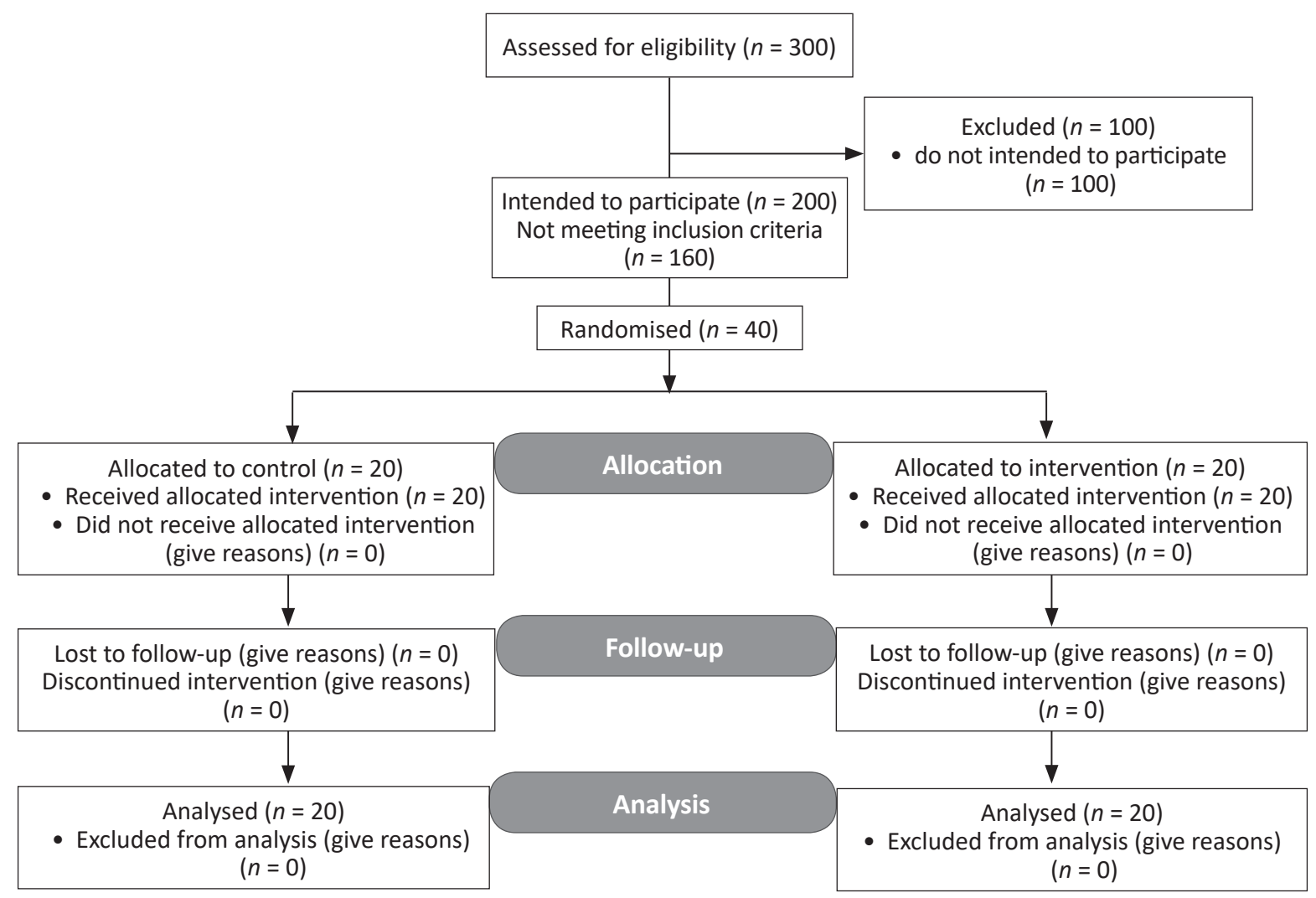

Figure 1. CONSORT 2010 flow diagram

The inclusion criteria of the study were as follows: scores of sexual satisfaction questionnaire and emotional quotient questionnaire lower than 75 and 270, respectively, higher than high school diploma education, experiencing at least one year of menopause, normal menopause, no drug addiction, no occurrence of stressful events in the last 6 months (severe and incurable disease, death of children or immediate family, accident, etc.), no use of medicines affecting sexual performance (antiseizure medications, drugs, anticholinergics, antihistamines, fat burners, progesterone, digoxin, cimetidine, psychedelic drugs and $\mathrm{H}_{2}$-receptor antagonists) and no diseases affecting sexual activities (thyroid, cancer, diabetics, blood pressure, pulmonary heart disease, trauma, surgery on genitals, infectious disease, depression, adrenal gland disorder, vasculitis and central nervous system disorders). In cases when the person avoided cooperating with the counsellor, did not intend to continue participating in the research, did not attend the sessions for at least two sessions consecutively or in the occurrence of stressful events to the women and family during the study, they were excluded from the study.

\section{Measurement tools}

In order to measure the variables of sexual satisfaction and the emotional quotient, Larson sexual satisfaction questionnaire and Bar-On's emotional quotient questionnaire were used before intervention in the study. Bar-On's emotional quotient questionnaire was standardised as a 90-question version in Persian. The minimum score of the questionnaire was 90 , and the maximum score was 450 . This questionnaire includes 15 sub-scales that were categorised into 5 general areas, i.e. intrapersonal relationship, interpersonal relationship, adaptability, stress management and general mood. Scoring the choices was arranged according to the Likert scale from 5 to 1 , and in some questions with negative content, the scoring was in reverse order (from 1 to 5 ). The score of each sub-scale was calculated by adding the scores of its 6 questions, and the overall test score was calculated by adding the scores of the 15 sub-scales. The minimum and maximum scores in each sub-scale were as follows: problem solving (13-30), happiness (12-30), independence (13-25), mental pressure tolerance (10-28), self-actualisation (11-30), emotional self-awareness (12-30), realism (8-30), interpersonal relationships (10-30), optimism (13-27), self-esteem (11-29), impulse control (8-29), flexibility (9-28), accountability (19-30), empathy (19-30) and self-assertiveness (11-28) [20].

The other questionnaire was the Larson sexual satisfaction questionnaire, and its validity and reliability were reported, respectively, as 0.90 and 0.86 in a research conducted by Shams (2001). This questionnaire included 25 phrases, and its five-choice answers were arranged according to the Likert scale from 1 to 5 . The minimum score was 25 , and the maximum score was 125 . Scores $25-50$ indicated sexual dissatisfaction, 51-75 low sexual satisfaction, 76-100 medium sexual satisfaction, and 101-125 indicated high sexual satisfaction.

\section{Statistical methods}

After collecting and coding, the data was entered into SPSS software version 23. For inferential statistics, in order to compare means, the $t$-test, chi-square test were used for demography analysis (Table 1 ), and the Mann-Whitney $U$ test and Wilcoxon Sign test were used for main outcome variables (Table 2, 3).

\section{Ethical consideration}

This research was accepted by the ethical committee of the Arak University of Medical Sciences under the code: IR.ARAKMU. REC.1396.3136. 


\begin{tabular}{|c|c|c|c|c|}
\hline & & Intervention & Control & \\
\hline \multicolumn{2}{|l|}{ Variable } & mean \pm SD & mean \pm SD & $p^{*}$ \\
\hline \multicolumn{2}{|l|}{ Age } & $57.95( \pm 6.32)$ & $54.4( \pm 4.79)$ & 0.06 \\
\hline \multicolumn{2}{|l|}{ Marriage duration } & $33.45( \pm 9.26)$ & $29.16( \pm 5.28)$ & 0.08 \\
\hline \multicolumn{2}{|c|}{ Age of last menstrual period } & $47.82( \pm 5.35)$ & $47.65( \pm 4.18)$ & 0.18 \\
\hline \multicolumn{2}{|c|}{ Number of pregnancies } & $2.63( \pm 1.01)$ & $2.70( \pm 0.92)$ & 0.26 \\
\hline \multicolumn{2}{|l|}{ Number of deliveries } & $2.67( \pm 0.9)$ & $2.55( \pm 0.51)$ & 0.50 \\
\hline \multirow{3}{*}{$\begin{array}{l}\text { Education } \\
n(\%)\end{array}$} & diploma & $65 \%(13)$ & $45 \%(9)$ & \multirow{3}{*}{0.39} \\
\hline & Associate's degree & $25 \%(5)$ & $45 \%(9)$ & \\
\hline & Bachelor's degree & $10 \%(2)$ & $2 \%(10)$ & \\
\hline \multirow{4}{*}{$\begin{array}{l}\text { Husband's education } \\
n(\%)\end{array}$} & lower diploma & $25 \%(5)$ & $15 \%(3)$ & \multirow{4}{*}{0.78} \\
\hline & diploma & $30 \%(6)$ & $25 \%(5)$ & \\
\hline & Associate's degree & $20 \%(4)$ & $30 \%(6)$ & \\
\hline & Bachelor's degree & $25 \%(5)$ & $30 \%(6)$ & \\
\hline \multirow{3}{*}{$\begin{array}{l}\text { Woman's job } \\
n(\%)\end{array}$} & employed & $50 \%(10)$ & $30 \%(6)$ & \multirow[t]{3}{*}{0.42} \\
\hline & housewife & $30 \%(6)$ & $45 \%(9)$ & \\
\hline & self-employed & $20 \%(4)$ & $25 \%(5)$ & \\
\hline \multirow[t]{2}{*}{ Husband's job $n(\%)$} & employed & $55 \%(11)$ & $60 \%(12)$ & \multirow[t]{2}{*}{0.74} \\
\hline & self-employed & $45(9)$ & $40 \%(8)$ & \\
\hline
\end{tabular}

* chi-square test.

\begin{tabular}{|l|l|l|l|l|}
\hline \multicolumn{5}{|c|}{ Table 2. Mean sexual satisfaction in intervention and control groups before and after the study } \\
\hline \multirow{2}{*}{ Sexual satisfaction } & & Before & After & $\boldsymbol{p}^{*}$ \\
\hline & Control & $68( \pm 12.2)$ & $69.8( \pm 11.8)$ & 0.30 \\
\cline { 2 - 5 } & Intervention & $67.3( \pm 13)$ & $78.3( \pm 14.8)$ & 0.001 \\
\hline$p^{\bullet}$ & & 0.8 & 0.05 & \\
\hline
\end{tabular}

*Wilcoxon; $\cdot$ Mann-Whitney U test.

\begin{tabular}{|c|c|c|c|c|c|}
\hline \multirow[t]{2}{*}{ Emotional quotient components } & \multicolumn{2}{|l|}{ Intervention } & \multicolumn{2}{|l|}{ Control } & \multirow[t]{2}{*}{$p^{*}$} \\
\hline & Before & After* & Before & After* & \\
\hline Intrapersonal relationship & $83( \pm 11.7)$ & $98.62( \pm 12.3)$ & $84.1( \pm 10.08)$ & $83.6( \pm 10.7)$ & 0.001 \\
\hline$p$-value ${ }^{\bullet}$ & \multicolumn{2}{|l|}{0.00} & \multicolumn{2}{|l|}{0.30} & \\
\hline Interpersonal relationship & $50.3( \pm 11.22)$ & $54.5( \pm 9.7)$ & $51.4( \pm 8.2)$ & $51(8.1)$ & 0.001 \\
\hline$p$-value ${ }^{\bullet}$ & \multicolumn{2}{|l|}{0.007} & \multicolumn{2}{|l|}{0.31} & \\
\hline Adaptability & $41.2( \pm 6.19)$ & $93.5( \pm 12.9)$ & $41.8( \pm 5.64)$ & $41.5( \pm 5.9)$ & 0.001 \\
\hline$p$-value ${ }^{\bullet}$ & \multicolumn{2}{|l|}{0.001} & \multicolumn{2}{|l|}{0.30} & \\
\hline Stress & $17.9( \pm 6.32)$ & $57.35( \pm 12.3)$ & $18.1( \pm 4.51)$ & $17.82( \pm 4.3)$ & 0.001 \\
\hline$p$-value• & \multicolumn{2}{|l|}{0.001} & \multicolumn{2}{|l|}{0.32} & \\
\hline General mood & $19( \pm 4.88)$ & $47.5( \pm 6.7)$ & $20.4( \pm 4.24)$ & $20.2( \pm 4.21)$ & 0.001 \\
\hline$p$-value ${ }^{\bullet}$ & \multicolumn{2}{|l|}{0.001} & \multicolumn{2}{|l|}{0.30} & \\
\hline
\end{tabular}

*Wilcoxon; $・$ Mann-Whitney $\mathrm{U}$ test.

\section{Results}

This study was conducted on 40 postmenopausal women in two groups (intervention and control). The average age of participants in the control group was $54.4 \pm 4.79$, and in intervention group, it was $57.95 \pm 6.32$. Furthermore, the average age in the last menstrual period in the control group was $47.65 \pm$ 4.18 , and in intervention group, it was $47.82 \pm 5.35$. According to Table 1, in the intervention and control groups, there was no significant difference in terms of demographic variables. Other research variables are presented in Tables 2. Table 2 showed that there was a significant difference between the sexual sat- isfaction scores in the intervention group after the study. The significance level in this study was equal to 0.05 .

After the intervention, the mean emotional quotient in the intervention group was significantly higher than those of the control group (Table 3 ).

\section{Discussion}

Although the emotional quotient increased significantly after intervention in the control group, sexual satisfaction was not significant. Sexual satisfaction after counselling only increased significantly in the intervention group after intervention com- 
pared to before intervention. Rellini et al. investigated the relationship between sexual problems and low or lack of emotional regulation in trauma-exposed cigarette smokers. Since low or lack of emotional regulation has a negative effect on interpersonal relationships, especially the relationship with the spouse, and trauma-exposed people have problems with controlling emotions and may experience sexual dissatisfaction, this study indicated that low emotional regulation is related to sexual dissatisfaction, especially in trauma-exposed cigarette smokers [19].

In the study conducted by Heidari et al., the relationship of the emotional quotient on the marital satisfaction of menopausal women was investigated. According to the results, couples with a high emotional quotient understand each other better, can manage their behaviours and are able to control their emotions, as well as the emotions of their spouses [21].

In the study conducted by Omidi et al., marital satisfaction was predicted according to the emotional quotient. They concluded that the emotional quotient can predict marital satisfaction and stated that close relationships require communication skills, such as empathy and understanding of the spouse's needs. Furthermore, couples with greater understanding who know how to express their emotions to other people can avoid misunderstandings, especially in a sexual relationship [22]. One of the components of the emotional quotient is social interactions, and in the present study, the interpersonal relationships of menopausal women who had received counselling based on emotional quotient increased.

In this study, women's self-awareness and empathy increased significantly during the emotional regulation counselling. Since a part of sexual satisfaction relates to expressing emotions, criticisms and suggestion to the spouse regarding sexual matters, women's awareness about their husband's sexual needs and their attempt to be in tune with their husband in a sexual relationship through emotional quotient skills helped the counsellor to increase sexual satisfaction. Therefore, in this research, it was found that by teaching the skills of self-awareness and self-assertiveness to menopausal women, they could overcome their sense of shame and improve their sexual satisfaction.

In the present study, the counsellor attempted to increase women's sexual satisfaction by teaching how to express needs and emotions to the husband through emotional quotient skills, such as self-awareness, empathy, interpersonal relationships and self-assertiveness. In fact, people experience sexual satisfaction when both sides are aware of their spouse's sexual needs, and this is achieved through emotional self-disclosure. Sayyadi et al. showed that higher sexual assertiveness increased positive feelings towards the other spouse [23].

Rehman et al. investigated the relationship between the sexual self-disclosure and sexual satisfaction of 91 couples. They found that sexual self-disclosure can lead to sexual satisfaction only in men [24].

It seems that in this study, emotional regulation skills could not improve the sexual satisfaction of menopausal women. However, due to lack of similar interventional studies in this field, investigating the effect of emotional regulation counselling on sexual satisfaction of menopausal women requires further studies.

\section{Limitations of the study}

It should be noted that this research had limitations, such as lack of control confounder variables (marital satisfaction, partner knowledge about sexual relationship) and lack of follow-up in the long term.

Another limitation of this study is that the intervention was conducted on women who were educated. Therefore, women with lower education were not included in the study. Thus, this study could not be generalised to all menopause women. It is recommended that, in future studies, some individual or collective sessions should be held for menopausal women and their husbands so that these couples can benefit from emotional regulation counselling, since sexual satisfaction and emotional regulation involves a two-way relationship.

\section{Conclusions}

Although emotional regulation can be considered as an appropriate method to improve sexual satisfaction in these women, more study should be done in the future.

Acknowledgments. This research was approved by the Research Committee. We would like to thank the research deputy of Arak University of Medical Sciences, who collaborated in the approval of the project. Thanks also to the participants for giving their time to this study.

Source of funding: This work was funded from the authors' own resources.

Conflicts of interest: The authors declare no conflicts of interest.

\section{References}

1. Novak E. Berek \& Novak's gynecology. 15th ed. Philadelphia: Lippincott Williams \& Wilkins; 2012.

2. World Health Organization. Women, ageing and health: a framework for action. Focus on gender. Geneva: WHO; 2007. Available from URL: https://www.who.int/ageing/publications/Women-ageing-health-lowres.pdf.

3. Lam WWT, Fielding R. Is self-efficacy a predictor of short-term post-surgical adjustment among chinese women with breast cancer? Psychooncology 2007; 16(7): 651-659.

4. Susło R, Paplicki M, Dopierała K, et al. Fostering digital literacy in the elderly as a means to secure their health needs and human rights in the reality of the twenty-first century. Fam Med Prim Care Rev 2018; 20(3): 271-275.

5. Davison SL, Bell RJ, LaChina M, et al. Sexual function in well women: stratification by sexual satisfaction, hormone use, and menopause status. J Sex Med 2008; 5: 1214-1222.

6. Nazarpour S, Simbar M, Tehrani FR. Factors affecting sexual function in menopause: a review article. Taiwan J Obstet Gynecol 2016; 55: 480-487.

7. Dundon $\mathrm{CM}$, Rellini AH. More than sexual function: predictors of sexual satisfaction in a sample of women age 40-70. Taiwan J Obstet Gynecol 2010; 7: 896-904.

8. Lawrance KA, Byers ES. Sexual satisfaction in long-term heterosexual relationships: the interpersonal exchange model of sexual satisfaction. Pers Relatsh 1995; 2: 267-285.

9. Previti D, Amato PR. Is infidelity a cause or a consequence of poor marital quality? J Soc Pers Relat 2004; 21: 217-230.

10. Rahmani A, Alahgholi L, Khuee EM. How does sexual satisfaction relate to marital satisfaction among Iranians? Int J Gynaecol Obstet 2009; 107: S558-S559.

11. Aghdam NSZ, Chizari M, Vakilian K, et al. Predictors of female sexual self-disclosure in Tehran. JMUMS 2017; 26: 357-361.

12. Abedi $\mathrm{P}$, Jafarzadeh $\mathrm{M}, \mathrm{Abbaspoor} Z$, et al. Education for sexual satisfaction promotion in postmenopausal women: individual or group education? IJOGI 2017; 20: 16-23. 
13. Karimi A, Dadgar S, Afiat M, et al. The effect of sexual health education on couples' sexual satisfaction. IJOG/ 2013; 15: 23-30.

14. Lopes PN, Salovey P, Côté S, et al. Emotion regulation abilities and the quality of social interaction. Emotion 2005; 5(1): $113-118$.

15. Thompson RA. Emotional regulation and emotional development. Educ Psychol Rev 1991; 3: 269-307.

16. Hill CL, Updegraff JA. Mindfulness and its relationship to emotional regulation. Emotion 2012; 12(1): 81-90.

17. Bloch L, Haase CM, Levenson RW. Emotion regulation predicts marital satisfaction: more than a wives' tale. Emotion 2014; 14(1): 130-144.

18. Cordova JV, Gee CB, Warren LZ. Emotional skillfulness in marriage: intimacy as a mediator of the relationship between emotional skillfulness and marital satisfaction. J Soc Clin Psychol 2005; 24: 218-235.

19. Rellini AH, Vujanovic AA, Zvolensky MJ. Emotional dysregulation: concurrent relation to sexual problems among trauma-exposed adult cigarette smokers. J Sex Marital Ther 2010; 36: 137-153.

20. Eslami AA, Hasanzadeh A, Jamshidi F. The relationship between emotional intelligence health and marital satisfaction: a comparative study. J Educ Health Promot 2014; 3, doi: 10.4103/2277-9531.127616.

21. Heidari M, Shahbazi S, Ghafourifard M, et al. Prediction of marital satisfaction based on emotional intelligence in postmenopausal women. J Menopausal Med 2017; 23(3): 196-201.

22. Omidi A, Talighi E. Prediction couple's marital satisfaction based on emotion regulation. Int J Educ Psychol Res 2017; 3: 157-162

23. Sayyadi F, Golmakani N, Ebrahimi M, et al. The relationship between sexual assertiveness and positive feelings towards spouse in married women. JMRH 2018; 6(3): 1305-1310.

24. Rehman US, Rellini AH, Fallis E. The importance of sexual self-disclosure to sexual satisfaction and functioning in committed relationships. J Sex Med 2011; 8: 3108-3115.

Tables: 3

Figures: 1

References: 24

Received: 23.04 .2019

Reviewed: 13.05 .2019

Accepted: 3.07.2019

Address for correspondence:

Katayon Vakilian, PhD, Assoc. Prof.

Peyambare Azam Campus

Arak University of Medical Sciences

Sardasht street

Arak

Iran

Tel.: +98 8634173524

E-mail: dr.kvakilian@arakmu.ac.ir 